\title{
A class of new modulus-based matrix splitting methods for linear complementarity problem
}

\author{
Shi-Liang $\mathrm{Wu}^{1}$ and Cui-Xia $\mathrm{Li}^{1}$ \\ ${ }^{1}$ Yunnan Normal University
}

September 16, 2020

\begin{abstract}
In this paper, to economically and fast solve the linear complementarity problem, based on a new equivalent fixed-point form of the linear complementarity problem, we establish a class of new modulus-based matrix splitting methods, which is different from the previously published works. Some sufficient conditions to guarantee the convergence of this new iteration method are presented. Numerical examples are offered to show the efficacy of this new iteration method. Moreover, the comparisons on numerical results show the computational efficiency of this new iteration method advantages over the corresponding modulus method, the modified modulus method and the modulus-based Gauss-Seidel method.
\end{abstract}

\section{Hosted file}

A class of new modulus-based matrix splitting methods for linear complementarity problem.pdf available at https : //authorea.com/users/359652/articles/481489-a-class-of-new-modulus-basedmatrix-splitting-methods-for-linear-complementarity-problem 